\title{
Combined iDPC and EELS analyses for quantifying oxygen vacancy concentration in LSMO
}

Aubrey Penn ${ }^{1}$, Sanaz Koohfar ${ }^{1}$, Divine Kumah ${ }^{1}$ and James LeBeau ${ }^{2}$

${ }^{1}$ North Carolina State University, United States, ${ }^{2}$ Massachusetts Institute of Technology, Cambridge, Massachusetts, United States

Thin film $\mathrm{La}_{0.7} \mathrm{Sr}_{0.3} \mathrm{MnO}_{3}$ (LSMO) has garnered much attention for use in magnetic memory and spintronic devices due to its colossal magnetoresistance and room temperature ferromagnetism [1]. LSMO's tunable magnetic character has also prompted fundamental studies on interfacial electronic structure engineering via substrate choice and complex heterostructures to control the strain state and octahedral distortion [2]. In many cases, scanning transmission electron microscopy (STEM) is used for studying thin films because of its ability to expose properties buried beneath the film surface at the atomic scale and in real space. A recent study of multilayered LSMO-La0.7 $\mathrm{Sr}_{0.3} \mathrm{CrO}_{3}$ heterostructures, however, uncovered a discrepancy in lattice parameters measured with STEM and X-ray diffraction (XRD) due to preferential oxygen vacancy formation in LSMO during STEM sample preparation [3]. In particular, samples for electron microscopy are often prepared by mechanical wedge polishing or focused ion beam milling, where both techniques introduce defects in the material due to harsh preparation environments. Even without external stimulus, LSMO tends to form oxygen vacancies due to Sr-doping, with vacancies forming on the apical positions on the perovskite octahedra [4]. Deciphering the origins of charge variation with electron microscopy thus requires consideration of both the growth conditions and sample preparation.

In this presentation, we discuss the effects of sample preparation on LSMO thin films using atomic resolution electron energy loss spectroscopy (EELS), annular dark field (ADF), and integrated differential phase contrast (iDPC) STEM. ADF and iDPC images, Figure 1(a, b), show that the LSMO film with average out-of-plane lattice parameter $3.87 \AA$ is expanded relative to the XRD-determined lattice parameter of $3.83 \AA$. We show that the direct visualization and quantification of oxygen vacancies in apical positions of LSMO octahedra is possible with iDPC and multislice image simulations. For example, Figure 1(c) shows layer-resolved atom column intensities with dimmer oxygen columns lying in the same plane as the bright La/Sr columns, indicating oxygen vacancy ordering in the predicted layers. Figure 1(d) shows experimental and simulated iDPC images of stoichiometric and oxygen deficient LSMO with oxygen vacancies placed at apical positions. From simulated oxygen column intensities, an estimated oxygen vacancy concentration of $\delta=0.125$ is reported, with stoichiometry denoted by $\mathrm{La}_{0.7} \mathrm{Sr}_{0.3} \mathrm{MnO}_{3}-\delta$. We additionally present an attempt to mitigate the oxygen vacancy formation during sample preparation by annealing prepared samples in oxygen. Examination of the EELS Mn and O core-loss edges, Figure 1(e), return no detectable change in oxygen stoichiometry, but rather evidence for change in oxygen coordination after annealing. Overall, we highlight the importance of combining multiple analysis techniques within and outside of STEM when making conclusions on a material's atomic structure and chemistry, especially in systems prone to defect formation. 
(a)

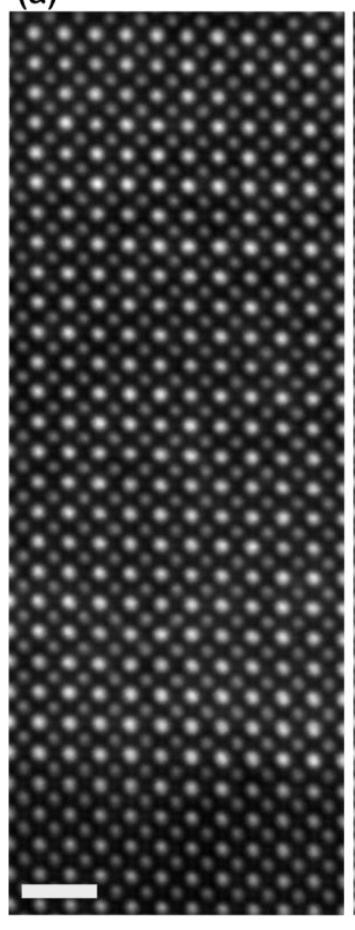

(b)

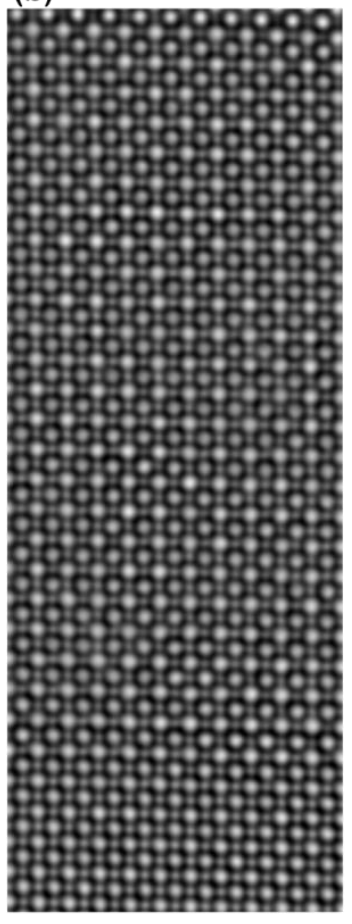

(c)

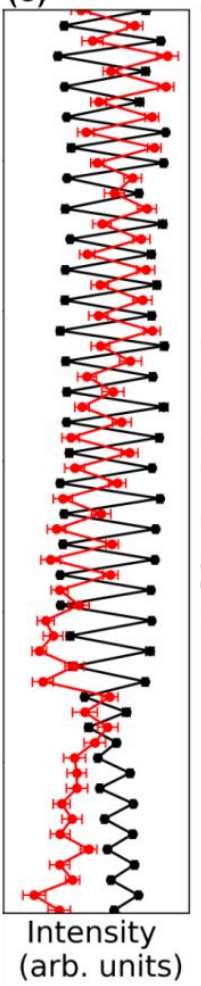

(d)

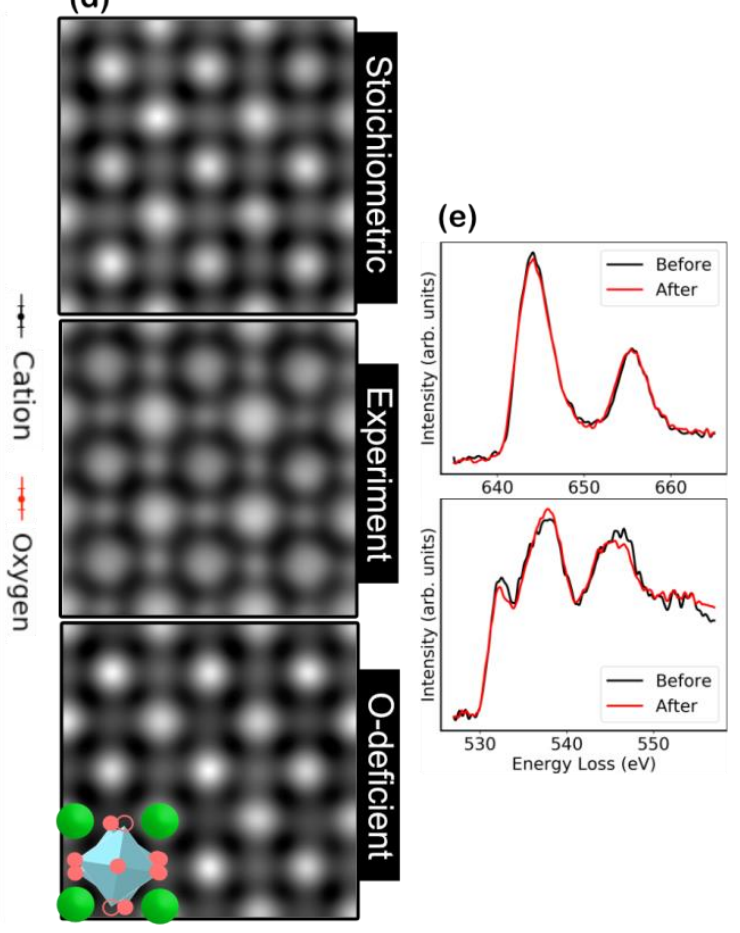

Figure 1. (a) ADF and (b) iDPC STEM images of LSMO on STO. The scale bar represents $1 \mathrm{~nm}$. (c) layer-resolved average atom column intensities (d) simulated and experimental images of LSMO with inset structure. Green, blue, and pink circles correspond to $\mathrm{La} / \mathrm{Sr}, \mathrm{Mn}$, and $\mathrm{O}$, respectively. Hollow circles correspond to apical positions where oxygen vacancies were included in simulations. (e) Mn L- and O Kedges before and after annealing the prepared sample.

References

1. Z. H. Xiong, D. Wu, Z. V. Vardeny, et al., Nature, 427 (2004) p. 821.

2. R. Peng, H. C. Xu, M. Xia, et al., Applied Physics Letters, 104 (2014), p. 081606.

3. A. N. Penn, S. Koohfar, D. P. Kumah, et al., AIP Advances, 10 (2020) p. 045113

4. S. Kobayashi, S. D. Findlay, N. Shibata, et al., Applied Physics Letters, 100 (2012). p. 193112

5. This work was supported by the National Science Foundation (DMR-1350273). The authors also acknowledge the Analytical Instrumentation Facility (AIF) at North Carolina State University, which is supported by the State of North Carolina and the National Science Foundation. 